\title{
Efectividad de una mezcla de cipermetrina y clorpirifós para el control de la mosca Haematobia irritans
}

\author{
Mixture of cipermetrina and clorpirifós to control Haematobia \\ irritans fly
}

\begin{abstract}
Gustavo López V,1* M.Sc, Cristiano Grisi do N, ${ }^{2}$ M.Sc, Diego González C, ${ }^{3}$ MV.
${ }^{1}$ Instituto Colombiano de Medicina Tropical CES. Medellín, Colombia ${ }^{2} O u r o$ Fino Animal Health Gerente de Marketing/Marketing Manager, Brasil. 32aboratorios Ourofino, Bogotá. *Correspondencia: gulova@ une.net.co
\end{abstract}

Recibido: Junio de 2010; Aceptado: Febrero de 2011.

\section{RESUMEN}

Objetivo. Determinar la eficacia de una mezcla de Cipermetrina y Clorpirifós contra la mosca de los cuernos Haematobia irritans. Materiales y métodos. Se utilizaron 24 novillas Holstein con infestación natural de moscas Haematobia irritans y se asignaron aleatoriamente a dos grupos, tratado y control, de 12 animales cada uno. El grupo tratado recibió el producto por aspersión en dilución 1:800, utilizando 1 litro por cada 100 kilos de peso. Se hicieron conteos de moscas antes del tratamiento y en los días 7, 14 y 21 postaplicación para evaluar la eficacia del producto. Resultados. Los porcentajes de eficacia del producto fueron de $89.9,97.4$ y $99.5 \%$, para los días 7,14 y 21 post-tratamiento, respectivamente. Conclusiones. La mezcla de Cipermetrina y Clorpirifós resultó altamente eficaz contra la mosca de los cuernos Haematobia irritans y surge como una nueva alternativa para el control del parásito, especialmente donde se presente resistencia a las diferentes moléculas que contienen los productos comerciales.

Palabras clave: Bovinos, ectoparasitosis, Holstein, moscas, tratamientos químicos. (Fuente: $C A B$ ) 


\section{ABSTRACT}

Objective. To determine the effect of a mixture of Cypermethrin and Chlorpiriphos on the control of the Haematobia irritans fly (horn fly). Materials and methods. A total of 24 Holstein heifers, naturally infested with Haematobia irritans flies, were randomly allocated into two Groups ( $n=12): 1$ ) Group Control and 2) Group Treated. The control group was not sprayed with any product and the treated group was sprayed with the product diluted (1:800), using 1 liter per 100 kilos of body weight. to evaluate the effect of the product. Flies were counts in each animal before treatment (day 0 ) and on days 7, 14 and 21 post application of the product. Total number of flies (\%) were compared between groups and between days. Results. Efficacy of the product were $89.9,97.4$ and $99.5 \%$, for days 7, 14 and 21 post-treatment, respectively. Conclusions. The mixture of Cypermethrin and Chlorpiriphos was highly effective against the horn fly and stands up as a new alternative for the control of the parasite, especially where resistance to different molecules of commercial products have occurred.

Key words: Bovines, chemical treatment, ectoparasitoses, flies, Holstein. (Source: CAB) .

\section{INTRODUCCIÓN}

En Colombia las pérdidas económicas ocasionadas por los ectoparásitos del ganado, moscas y garrapatas fueron estimadas a principios de esta década en más de \$ 76.000 millones anuales (1); sin embargo, en la actualidad la información sobre pérdidas económicas relacionadas con garrapatas y moscas es muy escasa y se requieren estudios que determinen las pérdidas para cada parásito y por regiones, por cuanto los estudios clásicos relacionados con aspectos económicos de garrapatas y moscas fueron realizados en climas templados y las poblaciones y costos de tratamientos han aumentado considerablemente (2).

En Colombia las moscas de mayor importancia veterinaria son conocidas como moscas picadoras o hematófagas y están representadas por dos especies: la mosca de los cuernos, Haematobia irritans (L., Díptera: Muscidae) y las mosca de los establos, Stomoxys calcitrans (L., Díptera: Muscidae) (1).

Haematobia irritans es una mosca hematófaga, parásito obligado que ocasiona cuantiosas pérdidas en la ganadería. Se ha calculado que por esta mosca se puede perder hasta un $14 \%$ del peso de novillos en pastoreo $(3,4)$.

La moca de los cuernos Haematobia irritans (L) afecta casi exclusivamente al ganado bovino y llegó a América procedente de
Europa entre 1884 y 1886 . En la actualidad presenta una amplia distribución en el continente americano (5).

Este ectoparásito además de ser vector de hemoparásitos altera el bienestar de los animales en la mayoría de pisos térmicos, situación que exige la aplicación permanente de productos químicos como estrategia principal de control, constituyéndose en costos exagerados para la canasta ganadera (1).

Haematobia irritans es una mosca pequeña con sus piezas bucales adaptadas para succionar sangre preferencialmente de bovinos, debido a sus requerimientos reproductivos y a la dependencia exclusiva de materia fecal bovina fresca para reproducirse, aunque en opinión de algunos autores (6), también puede alimentarse en otras especies animales como equinos, ovinos, caninos y hasta en humanos.

H. irritans se localiza de preferencia alrededor de los cuernos o en la parte dorsal y en épocas cálidas de brillo solar intenso o en épocas lluviosas su localización preferencial es la porción inferior del vientre (7).

El control de la mosca $H$. irritans en Colombia ha estado dirigido a la aplicación de productos fosforados y piretroides sintéticos mediante I sistemas de aspersión y con aretes u orejeras (8). En una encuesta realizada en Argentina 
en 806 predios de 11 provincias reportaron que el $94 \%$ de ellas utilizan mezclas de ivermectinas y organofosforados para el control de Haematobia irritans (9).

El control con base en insecticidas está íntimamente ligado al proceso de resistencia, fenómeno que ha sido creciente en muchas áreas de producción ganadera (10). Recientemente se han diagnosticado en diferentes paises poblaciones resistentes a productos organofosforados, carbamatos y piretroides $(4,10-12)$. Estudios realizados en México reportaron porcentajes de efectividad del 86 y $94 \%$ al utilizar Diazinón e Ivermectina inyectable respectivamente (13) aunque otros estudios reportan resultados muy variables de susceptibilidad de la mosca en pruebas in vitro (14). No se reportan en Colombia estudios sobre resistencia de la mosca de los cuernos Haematobia irritans a compuestos químicos.

Los sistemas utilizados hasta la fecha, basados en compuestos químicos, si bien ayudan a disminuir las altas infestaciones, no son sostenibles a mediano y largo plazo por la resistencia que se ha desarrollado a la mayoría de ellos, situación que requiere determinar la efectividad de otros compuestos que hagan más sostenible y económico el control (15). Ante la ineficacia de la mayoría de los compuestos químicos para el control de garrapatas, algunos investigadores mencionan el uso de mezclas de principios activos para buscar sinergismo entre ellos y eliminar las cepas resistentes (15).

No se conocen estudios en Colombia donde se utilicen mezclas de productos para el control y por esta razón se justificó estudiar la efectividad de la mezcla cipermetrina y clorpirifós en aspersión para el control de Haematobia irritans, la cual ha dado excelente resultado en el control de la garrapata común del ganado Rhipicephalus (Boophilus) microplus (16).

\section{MATERIALES Y MÉTODOS}

Compuesto químico. Para el estudio se utilizó una mezcla de Cipermetrina, Clorpirifós y Citronela de acuerdo con la siguiente concentración: Cipermetrina 15\% , Clorpiriphos $25 \%$ y Citronela $1 \%$. La dilución se hizo en la proporción de 1 parte del producto por 800 de agua según recomendación del laboratorio productor (Ourofino Ltda).

Animales experimentales. De un lote de 50 novillas Holstein de la hacienda El Puesto localizada en el municipio de la Ceja, departamento de Antioquia, se seleccionaron 24 y se dividieron en dos grupos tratado y control de 12 animales cada uno.

Inicialmente se reunieron los animales en un corral de cemento y después de dejarlos en reposo se realizó un recuento de moscas individualmente en todo el animal. Como el grado de infestación por moscas no se ajusta a una distribución normal, se conformaron los grupos aleatoriamente por conveniencia dejando los más parasitados en el grupo que recibiría el tratamiento.

Aplicación de los tratamientos. La aplicación del producto al grupo tratado se hizo utilizando una bomba de aspersión manual de palanca con una presión de 40 libras por pulgada cuadrada provista de boquilla de cono hueco y teniendo cuidado de humedecer muy bien todo el cuerpo de los animales, calculando 1 litro de la mezcla por cada 100 kilos de peso corporal.

En la aplicación del producto se hizo énfasis de iniciar de atrás hacia delante del animal y de abajo hacia arriba, haciendo énfasis en las orejas, tabla del cuello, papada, axilas, ubre, entrepierna, dorso, vientre y región perineal. Los recuentos de moscas se realizaron los días $0,7,14$ y 21 post tratamiento. El grupo control se dejó sin tratamiento

Después de la aplicación el grupo tratado se dejó en un potrero completamente separado y distante del grupo control por una cerca de alambre (cerca eléctrica) con el propósito de impedir que este último grupo estuviera en contacto con el tratado para evitar alteración de los resultados.

Determinación del porcentaje de eficacia. El porcentaje de eficacia fue determinado, relacionando el número de moscas, antes y después de la aplicación, 
en el grupo tratado, con el número de moscas antes y después de la aplicación, en el grupo control, para cada una de las evaluaciones post-tratamiento. Para el efecto se aplicó la fórmula descrita por Roulston (17) para este tipo de evaluaciones ectoparasitarias, así:

$\%$ de Supervivencia $=\{(a \times d) /(b \times c)\} \times$ 100 donde:

$\mathrm{a}=$ número de moscas en animales del testigo absoluto, en evaluaciones anteriores a la aplicación

$\mathrm{b}=$ número de moscas en animales del testigo absoluto, en evaluaciones posteriores a la aplicación

$c=$ Número de moscas en animales del grupo tratado en evaluaciones anteriores a la aplicación

$\mathrm{d}=$ Número de moscas en animales del grupo tratado en evaluaciones posteriores a la aplicación.

Eficacia $(\%)=100-\%$ de Supervivencia (17).

Aspectos éticos. Los procedimientos realizados para los tratamientos en el ganado bovino se rigió de acuerdo a la Ley 84 de 1989, capítulo VI, artículos 23 (literales a, b, c) y 24 del estatuto nacional de protección de los animales y que regula el uso de animales vivos en experimentos e investigación (18).

Asimismo para el cuidado, manejo y mantenimiento de animales se tuvieron en cuenta los protocolos y recomendaciones del IACUC (Institucional Animal Care and Use Committe) de la Universidad de Minnesota, en AVMA (American Veterinary Medical Association)(19), para la eutanasia y las recomendaciones sobre las buenas prácticas en la administración de sustancias y sangría de animales expuestas en el artículo EFPIA(European Federation of Pharmaceutical Industries Associations / ECVAM (European Center for the Validation of Alternative Methods) (20).

En esta investigación no se trabajó con muestras biológicas humanas por lo tanto no se determinaron las categorías de riesgo en humanos y se rigió de acuerdo a la resolución No. 008430 de 1993 emanada del Ministerio de Salud de la República de Colombia Titulo IV, en su Capitulo I, sobre la Bioseguridad de las Investigación y el manejo de material biológico que pueda contener microorganismos patógenos en los Artículos 63, 65, 66, 67 (literal c) y 69. La investigación debe cumplir con los aspectos éticos que figuran en la resolución 008430 de 1993 emanada del Ministerio de Salud en la cual en el Título II Capítulo 1, se establecen las normas éticas para la investigación en animales (21).

\section{RESULTADOS}

En las tablas 1 y 2 se observa el total de moscas en el grupo tratado y control antes del tratamiento y a los 7, 14 y 21 días después del tratamiento.

Tabla 1. Recuento de moscas Haematobia irritans en los grupos tratado y control.

\begin{tabular}{|c|c|c|c|c|c|c|c|c|c|}
\hline \multirow{2}{*}{$\begin{array}{l}\text { Grupo } \\
\text { Control }\end{array}$} & \multicolumn{4}{|c|}{$\mathrm{N}^{\circ}$ Moscas } & \multirow{2}{*}{$\begin{array}{l}\text { Grupo } \\
\text { tratado }\end{array}$} & \multicolumn{4}{|c|}{$\mathbf{N}^{\circ}$ Moscas } \\
\hline & Día 0 & 0 Día 7 & Día 14 & Día 21 & & Día 0 & 0 Día 7 & Día 14 & Día 21 \\
\hline 293 & 0 & 5 & 4 & 45 & 276 & 60 & 3 & 5 & 0 \\
\hline 290 & 8 & 8 & 7 & 95 & 246 & 60 & 0 & 2 & 0 \\
\hline 289 & 0 & 3 & 4 & 46 & 291 & 50 & 10 & 3 & 5 \\
\hline 296 & 0 & 2 & 2 & 47 & 269 & 69 & 2 & 5 & 0 \\
\hline 207 & 8 & 12 & 10 & 73 & 283 & 31 & 0 & 0 & 0 \\
\hline 292 & 10 & 6 & 7 & 91 & 273 & 39 & 5 & 0 & 0 \\
\hline 297 & 11 & 7 & 6 & 77 & 281 & 15 & 2 & 0 & 4 \\
\hline 294 & 7 & 6 & 6 & 40 & 277 & 60 & 2 & 0 & 0 \\
\hline 287 & 20 & 10 & 4 & 25 & 285 & 100 & 10 & 0 & 5 \\
\hline 216 & 6 & 7 & 8 & 31 & 284 & 54 & 8 & 0 & 0 \\
\hline 204 & 12 & 9 & 10 & 25 & 286 & 30 & 10 & 0 & 0 \\
\hline 200 & 56 & 58 & 69 & 72 & 279 & 94 & 4 & 0 & 0 \\
\hline
\end{tabular}

Tabla 2. Total de moscas Haematobia irritans en los grupos tratado y control durante el período experimental.

\begin{tabular}{ccc}
\hline Día experimental & $\begin{array}{c}\text { Población grupo } \\
\text { control }\end{array}$ & $\begin{array}{c}\text { Población grupo } \\
\text { tratado }\end{array}$ \\
\hline 0 & 156 & 662 \\
7 & 120 & 56 \\
14 & 137 & 15 \\
21 & 667 & 14 \\
\hline
\end{tabular}

Porcentaje de supervivencia para los días $7,14,21$, al comparar los grupos tratado y control (Figura 1) según la fórmula:

$(\mathrm{a} \times \mathrm{d}) /(\mathrm{b} \times \mathrm{c}) \times 100=$

Día 7: $(156 \times 56) /(120 \times 662) \times 100=10.1 \%$

Día 14: $156 \times 15) /(137 \times 662) \times 100=2.6 \%$ 
Día 21: $(156 \times 14) /(667 \times 662) \times 100=0.5 \%$

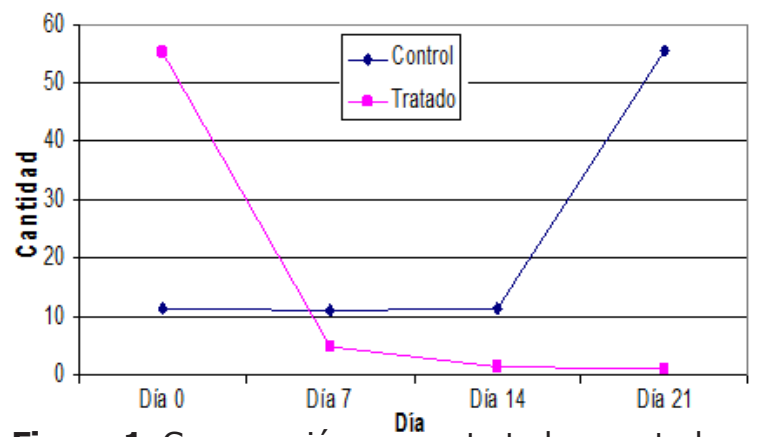

Figura 1. Comparación grúpos tratado y control.

De acuerdo con los resultados, el porcentaje de efectividad del producto para el día 7 fue del 89.9\%; para el día 14 el porcentaje de efectividad fue del $\mathbf{9 7 . 4 \%}$ y para el día 21 fue del $99.5 \%$.

\section{DISCUSIÓN}

Los resultados demuestran que en las condiciones del trabajo la mezcla de Cipermetrina y Clorpirifós tuvo una efectividad sobre la mosca de los cuernos Haematobia irritans del $89.9 \%$ a los 7 días de aplicado el producto, a los 14 días la efectividad fue del $97.4 \%$ y finalmente a los 21 de aplicado el producto la efectividad fue del $99.5 \%$.

Los resultados obtenidos al aplicar la mezcla de Cipermetrina y Clorpirifós sobre bovinos naturalmente infestados con Haemmatobia irrtans permitieron observar una disminución en el grado de infestación en más del $99 \%$ a los 7 días de haber aplicado el producto. Resultados similares de efectividad fueron reportados cuando se aplicaron formulaciones de mezclas de organofosforados y piretroides y de Cipermetrina y Clorpirifós respectivamente en cepas de garrapatas resistentes a cada producto individualmente $(22,23)$.

Infortunadamente no se tienen reportes de efectividad de la mezcla en el control de la mosca de los cuernos Haematobia irritans.

En conclusión, la prueba para determinar la eficacia del Cipermetrina y Clorpirifós sobre la mosca de los cuernos Haematobia irritans en el predio El Puesto, municipio de la Ceja, Antioquia, dio como resultado una efectividad del $99.5 \%$ a los 21 días de haber realizado el tratamiento. El porcentaje de efectividad a los 7 y 14 días pos tratamiento fue del 89.9 y $97.4 \%$ respectivamente. Este el primer reporte de la utilización de una mezcla de compuestos químicos para el control de la mosca Haematobia irritans. Sin embargo, con los resultados obtenidos, no podría concluirse sobre la presencia de una nueva formulación para el control de la mosca Haematobia irritans en Colombia y sería necesario replicar la investigación en diferentes pisos térmicos y con mayor número de repeticiones antes de generalizar recomendaciones para el control efectivo.

\section{REFERENCES}

1. Benavides OrtizE, Romero A. Consideraciones para el control integral de parásitos externos del ganado. Carta Fedegán 2001; 70: 64-85

2. Benavides Ortiz E. Control de las pérdidas ocasionadas por los parásitos del ganado. Anexo coleccionable "Manejo Integrado de Plagas y Enfermedades en explotaciones ganaderas 6". Carta Fedegan 2001; 69:52-63.

3. Kunz DH, Kemp DH. Insecticides and acaricides: resistance and environmental impact. Rev Sci Tech 1994; 13(4):1249- 1286.
4. Kunz DH, Ortiz EM, Fragosos SH. Status of Haematobia irritans ( Diptera: Muscidae) insecticide resistance in Northeastern Mexico. J Med Entomol 1994; 32(5):729-732.

5. Cicchino A, Abrahamovich A, Torres A, Núñez A, Prieto, O. Mosca de los cuernos, Haematobia irritans (Linnaeus, 1758), (Diptera: Muscidae). Contribuciones para su conocimiento en la Argentina. I: aspectos morfológicos básicos. Rev Med Vet 1983; 75:170-186.

6. Carballo $M$, Colombo A, Heizen $T$. Haematobia irritans, Mosca de la Paleta o Mosca de los Cuernos. Revista Veterinaria 1992; 28(117):5-13 
7. Williams RE, Towell C A. Comparison of insecticidal eartags and Ivermectinin a topical formulation for controlling Horn flies and Face flies (diptera: Muscidae) on pastured cattle. J Agr Entomol 1992; 9(4):283-288.

8. Martínez I F, Fragoso SH, Ortiz N A, Giles HI. Comparación entre el uso de uno y dos aretes mosquicidas impregnados con Ethion al 36\% en el control de la mosca de los cuernos Haematobia irritans resistente a los piretroides en ganado bovino. Acapulco, Guerrero, México: Memorias XXVI Congreso Nacional de Buiatría Julio 11 al 13; 2002.

9. Suárez VH, Castelli ME, Aguirre,DH, Alcaraz E, Cafrune M, Cetrá B et al. El uso de insecticidas para el control de Haematobia irritans (L): (Díptera:muscidae) en Argentina. INTA. Argentina. RIA 2006; 35(2):21-35.

10. Guglielmone AA, Castelli ME, Volpogni MM,Medus PD, Martins JR, Suárez VH et al. Toxicity of cypermethrin and diazinon to Haematobia irritans (Diptera:Muscidae) in its American southern range. Vet Parasitology 2001b; 101:67-73.

11. Barros AT, Guglielmoni AA, Martins JR. Mosca de los cuernos (Haematobia irritans): Control sustentable y resistencia a los insecticidas. Red Ectopar 2002; 1-10.

12. Barros AT, Ottea J, Sanson D, Foil LD. Horn fly (Diptera: Muscidae) resistance to organophosphate insecticides. Vet Parasitol 2001b; 96:243-256.

13. Maldonado $\mathrm{SE}$, Cadena $M$ JA, Sumano $L \mathrm{H}$, Martinez HA, Bermúdez VL. Evaluación de la eficacia del diazinón y la Ivermectina en el control de la mosca de los cuernos Haematobia irritans en bovinos en pastoreo en Tuxpan, Veracruz, México. Vet Méx 2003; 34(3): 261-267.

14. Maldonado SE, Apodaca SC, Sumano H I, Bermudez V I, García V Z, Gutiérrez O E. Susceptibilidad de Haematobia irritans de las zonas norte de Veracruz y centro de Nuevo león, Mexico a permetrina y diazinón. Vet Mex 2005; 36(2):217-227.
15. Guglielmone A A, Volponi $M M$, Scherling $N$, Muñoz Cobeñas $M$, Mangold A, Anziani OS et al. Chlorfenapyr ear tags to control Haematobia irritans (L) ( Diptera: Muscidae) on cattle. Vet Parasitol 2000; 93: 77-82.

16. López G, Grisi do Nascimento C, Gómez j, Valencia LA, Gonzalez CD. Evaluación de una mezcla de Cipermetrina + Clorpirifós sobre la garrapata Rhipicephalus(Boophilus) microplus en pruebas de campo y de laboratorio en el predio Román Gómez del politécnico de Marinilla, Antioquia. Rev CES 2009; 4(2):58-65.

17. Roulston WJ, Stone BF, Wilson JT, White L. Chemical control of organophosphorus and carbamate resistant strain of Boophilus microplus (Can.) from Queensland. Bull Ent Res 1.968; 58: 379-392.

18. Ley 84. Bogotá D.C. Colombia: Estatuto Nacional de Protección de los Animales. Congreso de Colombia; 1989.

19. Institucional Animal Care and Use Committe [en línea]. Minnesota: IACUC. 2005 [fecha de acceso junio de 2010]. URL disponible en: http://cflegacy.research.umn.edu/iacuc/

20. European Center for the Validation of Alternative Methods. [en línea]. Italy: Joint Research Centre, Institute for Health and Consumer Protection;2010.[fecha de acceso junio de 2010]. URL disponible en: http:// ecvam.jrc.it/

21. Resolución No 008430. Bogotá D.C. Colombia: Ministerio De Salud; 1993.

22. Betancourt A, Cassalett E, Escobar A, Uribe L. Experiencias con mezclas de compuestos acaricidas: susceptibilidad y alternativas de control de las garrapatas. Agricultura de las Américas. Primera entrega 1999; 272:3134.

23. DaCostaAJ. Relatorio Técnico. Cipermetrina+ Clorpirifós + Citronela en aspersión contra Boophilus microplus 2001. 